\title{
A note on Gevrey classes of functions comnected with Goursat problems
}

\author{
Jan Persson (*)
}

\begin{abstract}
Summary. - The Gevrey classes $G(\beta, 8, d)$ have been introduced in connection with non-linear local Goursat problems. A lemma is proved which shows that the definition of the class $G(\beta, \delta, d)$ can be simplified. It then follows that the results in two different papers on Goursat problems are compatible when specialized to formally identical cases.
\end{abstract}

The question if certain results for Gounsat problems in [1] and in [2] are compatible was raised in [2]. The definitions of the classes $G(\beta, \delta, d)$ and $G(0,0, d)$ in [1] use the existence of a certain real-valued function $b(y)$. It follows from a lemma below that the existence of the function by $(y)$ used in (1.4) in [1] follows from (1.3) in [1] and the continuity of the derivatives in. volved. The corresponding is true for (1.7) and (1.6). The function $b(y)$ is not used in the definition of the function class $G(\beta, d)$ in [2]. The lemma below shows that to every function of $G(\beta, d)$ there exists a function $b(y)$ satisfying an analogue of (1.4) in [1] and having the other wanted properties too. The lemma is easily extended to the conditions in [1]. It follows that (1.4) can be deleted in the definition of $G(\beta, \delta, d)$ without changing $G(\beta, \delta, d)$.

The same is true for $(1.7)$ and $G(0,0, d)$ in [1]. Therefore the results in [1] and [2] are compatible when specialized to formally indentical cases.

We shall use $(y, x) \in R^{2}$ as variables. We define $D_{x}=\partial / \partial x$ and $D_{y}=\mathrm{a} / \partial y$. We repeat definition 1 in $[2]$.

Dnfinition. - Let $d \geq 1$ be a real number, let $\beta$ be a positive integer, and let $u(x, y)$ be a realvalued function defined in some neighbourhood of the origin in $R^{2}$. The derivatives $D_{y}^{n} D_{x}^{\alpha} u$ are supposed to exist and to be continuous for $\eta \leq \beta$ and for all $\alpha$. If there exist constants $m>0$, and $r, 0<r<1$, such that for every pair $(\eta, \alpha)$ with $\eta+\alpha d \leq \beta$

$$
\left|D_{x}^{\xi} D_{y}^{n} D_{x}^{\alpha} u(y, x)\right| \leq m(2 r)^{-\xi \xi \xi \xi d}, \quad(y, x) \in V, \quad \text { all } \xi,
$$

then $u$ is said to belong to the function class $G(\beta, d, V)$. We define

$$
G(\beta, d)=\bigcup_{V}^{\cup} G(\beta, d, V)
$$

REMARK. - We use $2 r$ instead of $r$ in (1) for technical reasons.

(*) Entrata in Redazione il 17 ottobre 1968 . 
LEMMA. - If $u \in G, \beta, d, V)$ then (1) is true and there exists a function $b(y) \geq 0$ depending on $\lfloor y \mid$ only such that

$$
\begin{gathered}
\left|D_{x}^{\xi} D_{y}^{n} D_{x}^{\alpha} u(y, x)-D_{x}^{\xi} D_{y}^{n} D_{x}^{\alpha} u(0, x)\right| \leq m b(y) r^{-\xi \xi \xi d}, \quad(y, x) \in V, \quad(0, x) \in V, \\
\eta+\alpha d \leq \beta, \quad \text { all } \xi .
\end{gathered}
$$

The function $b(y)$ has the additional property

$$
y \rightarrow 0 \Rightarrow b(y) \rightarrow 0 \text { monotonically in }|y| \text {. }
$$

Proof of the Lemma. - The function $b(y)$ is defined by

$$
\begin{array}{cl}
\left.\left.b^{\prime} y\right)=\sup \mid D_{x}^{\xi} D_{y}^{n} D_{x}^{\alpha} u^{\prime} y^{\prime}, x\right)--D_{x}^{\xi} D_{y}^{n} D_{x}^{\alpha} u(0, x) \mid r^{\xi} \xi-\xi d, & \left|y^{\prime}\right| \leq|y|, \quad\left(y^{\prime}, x\right), \\
(y, x), \quad(0, x) \in V, \quad \eta+\alpha d \leq \beta, & \text { all } \xi .
\end{array}
$$

$V$ is supposed to be compact here. It follows from (1) that $b(y) \leq 2 m$.

In addition, for a given number $\varepsilon>0$ there exists a number $K>0$ such that when $\xi>K$

$$
\left|D_{x}^{\xi} D_{y}^{n} D_{x}^{\alpha} u\left(y^{\prime}, x\right)-D_{x}^{\xi} D_{y}^{n} D_{x}^{\alpha} u(0, x)\right| r^{\xi} \xi-\xi d \leq 2 m 2^{-\xi} \leq m 2^{1-K}<\varepsilon .
$$

There is only a finite number of derivatives corresponding to $(\xi, \eta, \alpha)$ with $\xi \leq K, \eta+\alpha d \leq \beta$. It follows from the uniform continuity of the derivatives of $u$ in $V$ that there exists a number $\delta>0$ such that

$$
\begin{aligned}
& \left|D_{x}^{\xi} D_{y}^{\eta} D_{x}^{x} u\left(y^{\prime}, x\right)-D_{x}^{\xi} D_{y}^{\eta} D_{x}^{\alpha} u(0, x)\right| r^{\xi}-\xi \xi^{\xi} d<\varepsilon, \\
& \left|y^{\prime}\right| \leq|y|<\delta, \quad \xi \leq K, \quad \eta+\alpha d \leq \beta .
\end{aligned}
$$

By that the lemma is proved since $b(y)<\varepsilon$ when $|y| \leq \delta$.

\section{REFERENCES}

[1] J. Persson, New proofs and generalizations of two theorems by Lednev for Goursat problems, Math. Ann. 178, 184.208 (1968).

[2] - - Exponential majorization applied to a non-linear Canchy (Goursat) problem for functions of Gevrey nature, Ann. Mat. pux. appl. LXXVII, 259.268 (1968). 